\title{
Hypofractionated radiotherapy induces miR-34a expression and enhances apoptosis in human nasopharyngeal carcinoma cells
}

\author{
ZHIXIONG LONG ${ }^{1,2^{*}}$, BIN WANG $^{3 *}$, DAN TAO $^{2 *}$, YING HUANG $^{2 *}$ and ZEZHANG TAO ${ }^{1}$ \\ ${ }^{1}$ Department of Otolaryngology-Head and Neck Surgery, Renmin Hospital of Wuhan University, Wuhan, \\ Hubei 430060; ${ }^{2}$ Department of Oncology, and ${ }^{3}$ Department of Otolaryngology (ENT), \\ The Fifth Hospital of Wuhan, Wuhan, Hubei 430051, P.R. China
}

Received April 26, 2014; Accepted September 10, 2014

DOI: $10.3892 /$ ijmm.2014.1937

\begin{abstract}
Nasopharyngeal carcinoma (NPC) is a relatively radiosensitive disease. However, the therapeutic effects of radiotherapy are not always satisfactory due to radioresistance. The hypofractionated schema is currently widely used in clinical practice. In the present study, we investigated the effects of hypofractionated radiotherapy on NPC cells and explored the mechanisms involved. In addition, we aimed to determine the role of miR-34a in the effects of hypofractionated radiotherapy and whether these effects occur in a p53-dependent manner. For this purpose, we used CNE1 and CNE2 NPC cells which were subjected to hyperfractionated and hypofractionated radiotherapy. The viability of the cells was measured by MTT assay and acridine orange (AO) and ethidium bromide (EB) staining was used to observe morphological changes. In addition, Annexin V-propidium iodide (PI) staining and flow cytometry were used to determine the number of apoptotic cells and mRNA and protein expression was measured by qPCR and western blot analysis, respectively. The results revealed that hypofractionated radiotherapy enhanced apoptosis and increased the expression of miR-34a and p53 in the NPC cells. In addition, it stimulated p53 promoter activity and downregulated the protein expression of c-Myc in the human NPC cells. Furthermore, the knockdown of miR-34a suppressed the growth inhibitory effects induced by hypofractionated radiotherapy. Thus, our results suggest that the enhanced apoptosis of NPC cells may be associated with the miR-34a-mediated suppression of c-Myc in a p53-dependent manner.
\end{abstract}

Correspondence to: Dr Zezhang Tao, Department of OtolaryngologyHead and Neck Surgery, Renmin Hospital of Wuhan University, 99 Zhangzhidong Road, Wuhan, Hubei 430060, P.R. China

E-mail: taozezhang@163.com

*Contributed equally

Key words: nasopharyngeal carcinoma, hypofractionated radiotherapy, miR-34a, p53

\section{Introduction}

Radiotherapy is the primary treatment modality for nasopharyngeal carcinoma (NPC). However, even if high dose-large field irradiation ( $>70 \mathrm{~Gy}$ ) is delivered, failure will occur in $30-50 \%$ of patients due to locally persistent or recurrent disease $(1,2)$. In addition, differences in radiosensitivity between tumor cells have become an important factor that affects the efficacy of radiotherapy for NPC.

With the increased usage of normal tissue sparing with highly conformal therapy, such as intensity-modulated radiation therapy (IMRT), it has become possible to treat patients with fewer treatments at a high dose/fraction. Approximately 20 years ago, hypofractionation was proposed as an alternative based on institutional historical experiences (3). However, there are still some concerns associated with these treatment options, such as the unpredictability of the complication probabilities for normal and tumor tissue. Recently, a promising technique, hypofractionated radiotherapy, was employed for the treatment of several types of cancer, including breast, prostate and lung cancer $(3,4)$. For NPC previously treated with linac-based hypofractionated stereotactic radiotherapy, the outcome has improved by decreasing local recurrence. Furthermore, for hypofractionated dose-painting intensity-modulated radiation therapy (DP-IMRT), the local control, freedom from distant metastases and overall survival are more favorably compared to other series of IMRT and chemotherapy (5). Since hypofractionated radiotherapy is an important alternative clinical radiotherapy for NPC, the elucidation of the relevant mechanisms of action would lay a solid theoretical foundation for the more extensive application of this treatment method in clinical practice.

MicroRNAs (miRNAs or miRs) are a class of small (19-22nt) non-protein-coding RNAs that negatively regulate genes by binding to the the $3^{\prime}$ untranslated region (3' UTR) of the target mRNA, inducing mRNA destabilization and/or translational inhibition through the RNA-induced silencing complex (6). It has been reported that the miR-17-92 cluster, when overexpressed in lymphomas and certain other solid tumors, induces a significant increase in the radioresistance of human mantle cell lymphoma cells (7). By contrast, miR-34a, which is generally expressed at lower levels in unfavorable tumors, functions as a potential tumor suppressor by inducing apoptosis in tumor 
cells (8). The miR-34 family is also significantly downregulated in NPC and targets the TGF-Wnt, apoptosis and survival pathways (IP3 signaling pathway, G1-S cell cycle progression and VEGF signaling pathway) that are elevated in NPC tissue $(9,10)$. miR-34a expression has been shown to increase upon treatment with radiation, subsequently resulting in cell cycle arrest and cell death in malignant mesothelioma cells (11). Moreover, the overexpression of miR-34a (through a p53-dependent manner) has been shown to significantly enhance the radiosensitivity of cancer cells, while the inhibition of miR-34a protects these cells from radiation injury (12).

In the present study, we investigated the effects of hypofractionated radiotherapy on the expression of p53, c-Myc, miR-34a and its target genes in human NPC cells.

\section{Materials and methods}

Cell culture and transfection. The human NPC cell lines, CNE1 and CNE2, were obtained from the American Type Culture Collection (ATCC, Manassas, VA, USA) and cultured in RPMI-1640 medium (Gibco, Grand Island, NY, USA) supplemented with 10\% FBS (HyClone, Logan, UT, USA), $100 \mathrm{U} / \mathrm{ml}$ penicillin and $100 \mu \mathrm{g} / \mathrm{ml}$ streptomycin (HyClone). The cell cultures were maintained at $37^{\circ} \mathrm{C}$, with $5 \% \mathrm{CO}_{2}$ under fully humidified conditions. Anti-miR-34a or negative control inhibitors (Guangzhou RiboBio Co., Ltd., Guangzhou, China) were transfected into confluent cells using Lipofectamine 2000 (Life Technologies Corp., Carlsbad, CA, USA). The cells were also transfected with pGL3/basic plasmid or pGL3/p53 promoter plasmid for measuring $\mathrm{p} 53$ promoter activity.

Irradiation. The cells were plated in 6-well plates $24 \mathrm{~h}$ prior to irradiation. In the relative hyperfractionated groups, the CNE1 and CNE2 cells were subsequently exposed to $10 \mathrm{~Gy} 6 \mathrm{MV}$ $\mathrm{X}$-rays in 5 fractions (2 Gy per fraction). In the hypofractionated groups, the CNE1 cells were exposed to 4 fractions of $6 \mathrm{MV}$ X-rays at 2.33 Gy per fraction, and the CNE2 cells were exposed to 4 fractions of $6 \mathrm{MV}$ X-rays at 2.43 Gy per fraction, maintaining the biological equivalent doses the same as those of the relative hyperfractionated groups.

Cell viability assay. Cell viability was measured by MTT colorimetric assay. The cells $\left(1 \times 10^{4}\right.$ in $100 \mu \mathrm{l}$ culture medium/well) were plated in 96-well microplates and were allowed to adhere overnight. Following exposure to X-rays, $20 \mu \mathrm{l}$ of MTT $(5 \mathrm{mg} / \mathrm{ml})$ were added to each well followed by incubation at $37^{\circ} \mathrm{C}$ for an additional $4 \mathrm{~h}$. Subsequently, the cell supernatants were discarded. MTT crystals were dissolved with DMSO and the absorbance was detected at $570 \mathrm{~nm}$ using a microplate reader (Tecan Ultra, Research Triangle Park, NC, USA). Cell viability (\%) was defined as the relative absorbance of the treated samples versus that of the untreated controls. All experiments were performed with 6 wells for each experiment and repeated at least 3 times.

Morphological observation of apoptotic cells. The acridine orange (AO) and ethidium bromide (EB) (both from Sigma) staining methods were applied to detect the changes in cell morphology. Briefly, the cells were harvested with $0.125 \%$ trypsin and $0.01 \%$ EDTA, resuspended in $95 \mathrm{ml}$ of minimal essential medium, and incubated with $5 \mathrm{ml}$ of $\mathrm{AO} / \mathrm{EB}$ staining solution (100 mg/l PBS of each dye) at room temperature for $15 \mathrm{~min}$. The cells were examined under a fluorescence microscope and photographed (Olympus, Tokyo, Japan). Viable cells were colored green with intact nuclei. Non-viable cells had bright orange chromatin. Apoptosis was demonstrated by the appearance of cell shrinkage with condensation and fragmentation of the nuclei. Necrotic cells appeared orange with a normal nuclear structure.

Quantification of apoptotic rates. The apoptotic ratios of the cells were determined by Annexin V-propidium iodide (PI) staining (BD Pharmingen, San Diego, CA, USA), using the Annexin V/PI Apoptosis Detection kit (BD Pharmingen) following the manufacturer's instructions. The cells were analyzed using a FACScan flow cytometer (BD Biosciences, San Jose, CA, USA). This method allows for distinguishing between living cells (Annexin $\mathrm{V}^{-} / \mathrm{PI}^{-}$), early apoptotic/primary apoptotic cells (Annexin $\mathrm{V}^{+} / \mathrm{PI}^{-}$), late apoptotic/secondary necrotic cells (Annexin $\mathrm{V}^{+} / \mathrm{PI}^{+}$) and necrotic cells (Annexin $\mathrm{V}^{-}$/ $\mathrm{PI}^{+}$). Data analysis was performed using CellQuest ${ }^{\mathrm{TM}}$ Pro Analysis software (BD Biosciences) and were expressed as a percentage of positive cells.

RNA isolation, reverse transcription and quantification of miRNA expression. Total RNA was isolated using TRIzol reagent (Invitrogen/Life Technologies Corp.), according to the manufacturer's instructions. The levels of mature miRNAs in the cell lines were determined using the Bulge-Loop miRNA qPCR Primer Set. After the cDNA was synthesized with a miRNA-specific stem-loop primer, qPCR was performed with specific primers (Guangzhou RiboBio Co., Ltd.). The miR-34a levels were normalized to those of U6 snRNA.

Western blot analysis. The cells were collected and lysed with $1 \mathrm{X}$ cell lysis buffer (Promega, Madison, WI, USA). Protein concentrations were measured by BCA protein assay (Pierce, Rockford, IL, USA). Equal amounts of total protein were boiled in sample buffer and separated by sodium dodecyl sulfate-polyacrylamide gel electrophoresis (SDS-PAGE). Following immunoblot analysis with antibodies [mouse antihuman GAPDH, rabbit anti-human p53, rabbit anti-human c-Myc (Cell Signaling Technology, Danvers, MA, USA)] as an alternative, immunoreactive bands were visualized using the specific HRP-conjugated secondary antibody, and the ECL substrate kit was used for the chemiluminescent detection of signals with autoradiography film (Amersham Biosciences, Piscataway, NJ, USA).

Dual-luciferase reporter assay for p53 promoter activity. The human 553 promoter-luciferase reporter was kindly provided by Dr Mingfang Wu (School of Medicine, Chung Shan Medical University, Taichung, Taiwan). Firefly and Renilla luciferase activities were consecutively measured according to the manufacturer's instructions (Promega). The luciferase activity was measured using a luminometer (Lumat LB 9507; Berthold Technologies, Bad Wildbad, Germany).

Statistical analysis. The results are presented as the means \pm standard error of the mean (SEM). Statistical signifi- 

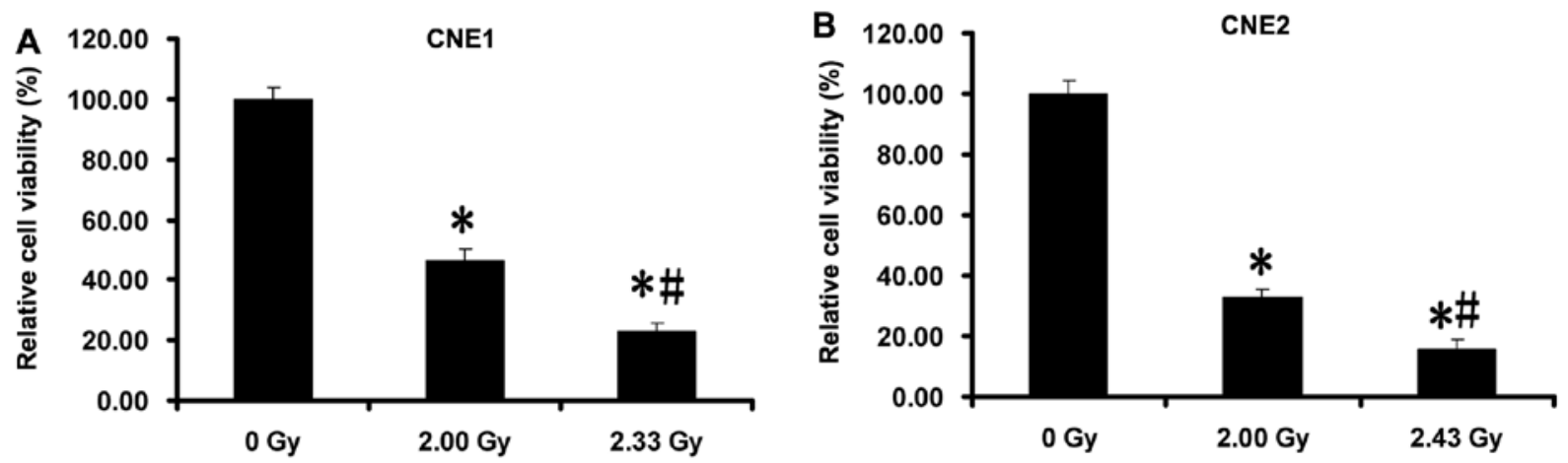

Figure 1. Hypofractionated radiotherapy enhances the growth inhibitory effects on nasopharyngeal carcinoma (NPC) cells. (A) CNE1 and (B) CNE2 cells were exposed to $2 \mathrm{~Gy} 6 \mathrm{MV}$ X-ray per fraction for 5 fractions in the relative hyperfractionated group, and (A) CNE1 cells were exposed to 2.33 Gy per fraction for 4 fractions in the hypofractionated group, while (B) CNE2 cells were exposed to 2.43 Gy per fraction for 4 fractions in the hypofractionated group. Cell viability was detected by MTT assay, as described in 'Materials and methods'. "P $<0.05$ vs. control. ${ }^{~} \mathrm{P}<0.05$ vs. relative hyperfractionated group. The results are the means $\pm \mathrm{SD}$ of triplicate experiments.

cance $(\mathrm{P}<0.05)$ was determined by the $\mathrm{t}$-test or one-way analysis of variance (ANOVA) followed by the assessment of the differences using SPSS 15.0 software (SPSS Inc., Chicago, IL, USA).

\section{Results}

Hypofractionated radiotherapy enhances the growth inhibitory effects on NPC cells. The MTT colorimetric assay indicated that, when compared with the untreated controls, the relative hyperfractionated radiotherapy inhibited the viability of the CNE1 and CNE2 NPC cells by $53.6 \pm 3.9$ and $67.3 \pm 2.9 \%$, respectively (Fig. 1). The hypofractionated radiotherapy inhibited the viability of the CNE1 and CNE2 NPC cells by $77.0 \pm 2.6$ and $84.2 \pm 3.1 \%$, respectively (Fig. 1); the inhibitory effects of hypofractionated radiotherapy on cell viability were significantly greater than those observed following hyperfractionated radiotherapy. These findings indicate that the hypofractionated schema can effectively enhance the growth inhibitory effects of radiotherapy on NPC cells in vitro.

Hypofractionated radiotherapy increases the apoptosis of NPC cells. In order to confirm whether apoptosis is involved in hypofractionated radiotherapy-induced cell death, we performed $\mathrm{AO}$ and $\mathrm{EB}$ staining in order to observe the changes occurring in cell morphology, as well as Annexin V-FITC and PI staining and flow cytometry to determinate the apoptotic cell ratios. As shown in Fig. 2A and B, compared with the relative hyperfractionated group, hypofractionated radiotherapy induced more extensive DNA strand breakage and shrunken nuclei, changes characteristic of apoptosis, in the CNE1 and CNE2 NPC cells. Annexin V-FITC and PI staining and flow cytometry further demonstrated that the apoptotic rates of the relative hyperfractionated radiotherapy-treated CNE1 and CNE2 cells were $51.6 \pm 1.9$ and $64.9 \pm 2.1 \%$, respectively (Fig. 2C and D). These rates significantly increased to $71.9 \pm 2.6$ and $82.7 \pm 2.7 \%$ in the CNE1 and CNE2 cells, respectively in the hypofractionated radiotherapy-treated group (Fig. 2C and D). In addition, compared with the apoptotic cells, the necrotic cells made up a very small portion of the dead cells. Taken together, these results demonstrate that hypofractionated radiotherapy inhibits NPC cell growth mainly by inducing cell apoptosis.

Hypofractionated radiotherapy upregulates miR-34a expression. Previous studies have demonstrated that miR-34a functions as an endogenous repressor of target genes by interfering with the expression of tumor-associated genes, and may be a novel indicator, mediator and target of radiation injury, radiosensitivity and radioprotection $(11,13,14)$. In the present study, we wished to determine whether the upregulation of miR-34a is associated with the increased apoptosis of NPC cells induced by hypofractionated radiotherapy. Our data indicated that the relative hyperfractionated radiotherapy slightly upregulated miR-34a expression in the CNE1 and CNE2 NPC cells, while hypofractionated radiotherapy significantly increased miR-34a expression (Fig. 3). To confirm the role of miR-34a in the effects of hypofractionated radiotherapy, we used anti-miR-34a inhibitor to suppress the expression of miR34at. As shown in Fig. 4, compared with the negative control inhibitor, the knockdown of miR-34a with anti-miR-34a inhibitor markedly suppressed the growth inhibitory effects induced by hypofractionated radiotherapy in the CNE1 and CNE2 NPC cells. These data verify that the upregulated expression of miR-34a indeed plays an important role in the effects of hypofractionated radiotherapy.

Hypofractionated radiotherapy enhances p53 gene transcription and protein expression, while c-Myc protein is downregulated. It has been demonstrated that the expression of the tumor suppressor, miR-34a, is regulated by p53 (15-17). In the present study, we found that, compared with the relative hyperfractionated group, hypofractionated radiotherapy induced a higher p53 protein expression in the CNE1 and CNE2 NPC cells, while c-Myc protein expression was markedly downregulated (Fig. 5). Furthermore, promoter reporter-luciferase assay revealed that compared with the relative hyperfractionated group, p53 promoter activity was significantly increased by hypofractionated radiotherapy (Fig. 6), indicating that both p53 gene transcription and protein expression were upregulated. These results suggest 
A CNE1

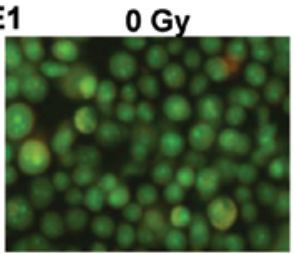

\section{B CNE2}

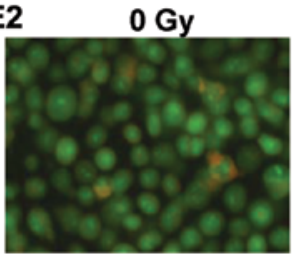

$2.0 \mathrm{~Gy}$

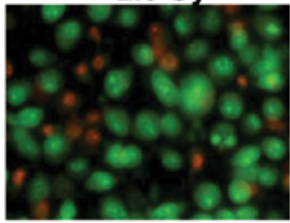

$2.0 \mathrm{~Gy}$

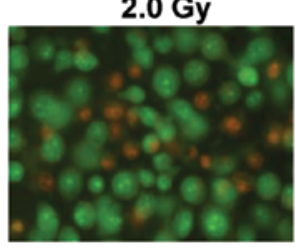

$2.33 \mathrm{~Gy}$

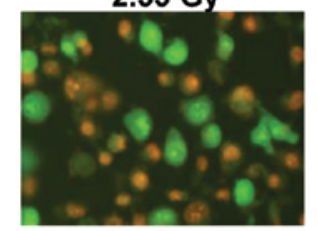

$2.43 \mathrm{~Gy}$

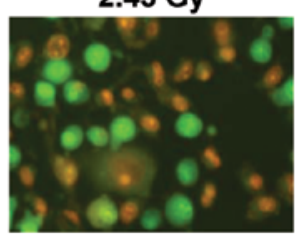

C

CNE1

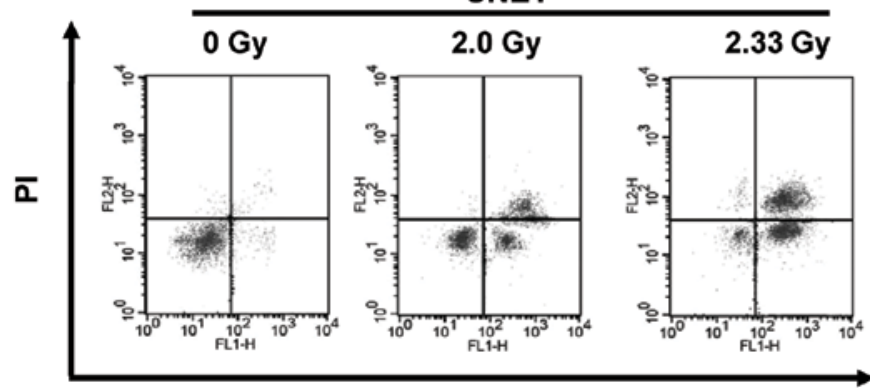

Annexin V

D

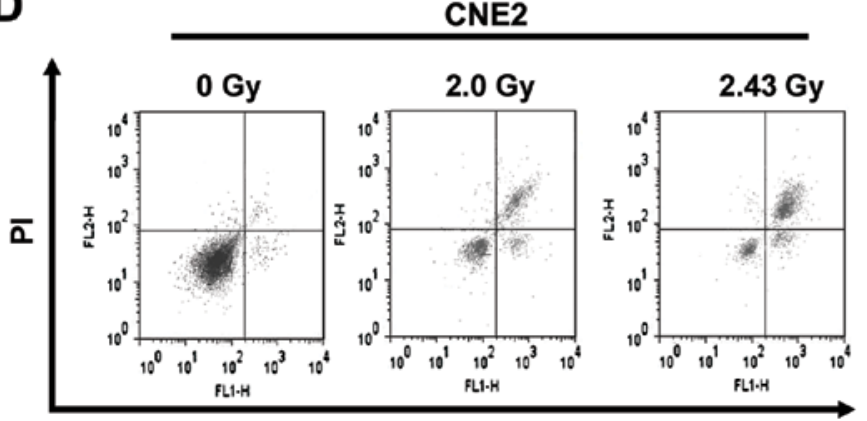

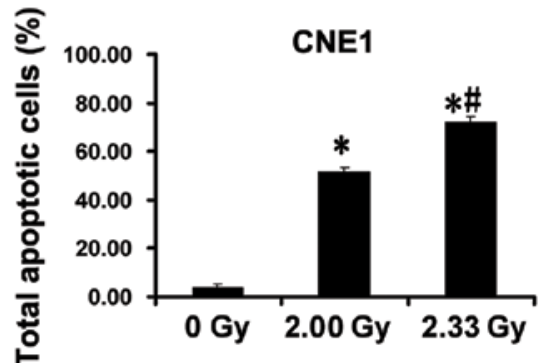

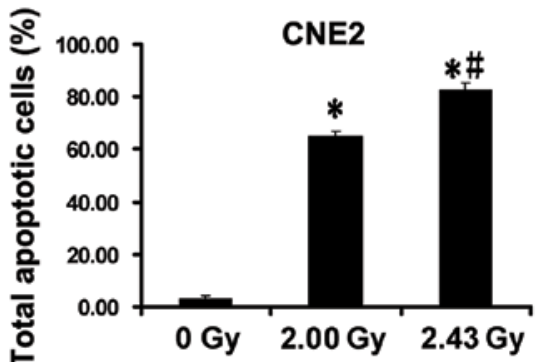

Annexin V

Figure 2. Hypofractionated radiotherapy increases the apoptosis of nasopharyngeal carcinoma (NPC) cells. (A and C) CNE1 and (B and D) CNE2 cells were exposed to $2 \mathrm{~Gy} 6 \mathrm{MV}$ X-ray per fraction for 5 fractions in the relative hyperfractionated group, and (A and C) CNE1 cells were exposed to 2.33 Gy per fraction for 4 fractions in the hypofractionated group, while (B and D) CNE2 cells were exposed to 2.43 Gy per fraction for 4 fractions in the hypofractionated group. The cells were then collected for acridine orange (AO)/ethidium bromide (EB) staining and florescence microscopy (A and B; magnification, x200), and Annexin V/PI double staining and flow cytometry $(\mathrm{C}$ and $\mathrm{D}) .{ }^{*} \mathrm{P}<0.05$ vs. control. ${ }^{*} \mathrm{P}<0.05$ vs. relative hyperfractionated group. The results are the means $\pm \mathrm{SD}$ of triplicate experiments.
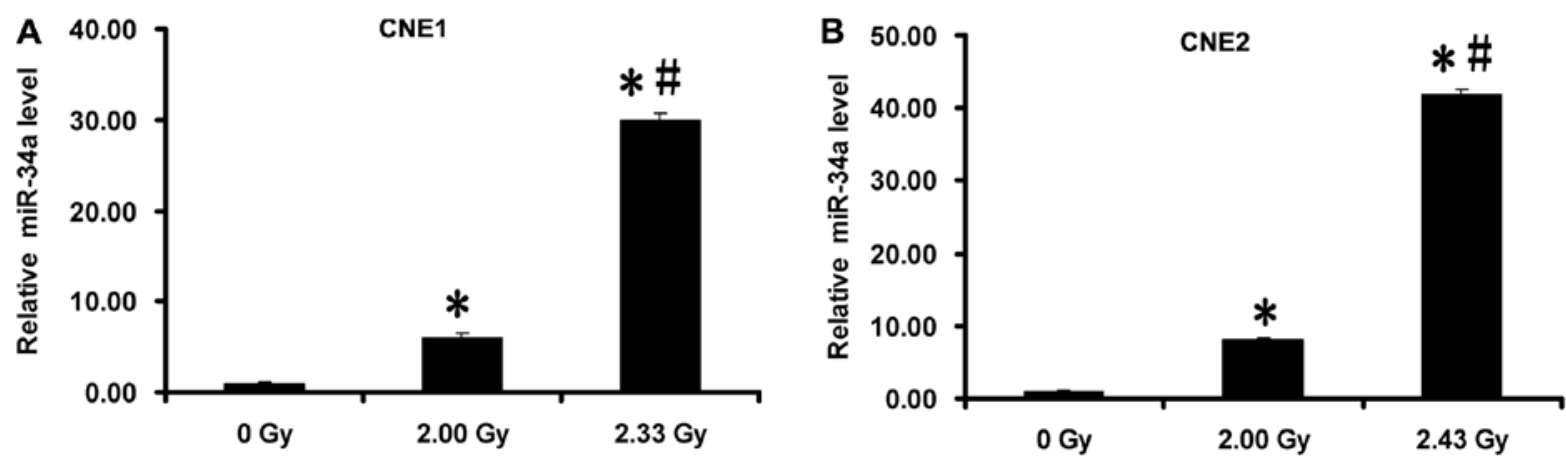

Figure 3. Hypofractionated radiotherapy upregulates miR-34a expression. (A) CNE1 and (B) CNE2 cells were exposed to 2 Gy 6 MV X-ray per fraction for 5 fractions in thye relative hyperfractionated group, and (A) CNE1 cells were exposed to 2.33 Gy per fraction for 4 fractions in the hypofractionated group, while (B) CNE2 cells were exposed to 2.43 Gy per fraction for 4 fractions in the hypofractionated group. miR-34a expression was detected by qPCR as described in 'Materials and methods'. " $\mathrm{P}<0.05$ vs. control. " $\mathrm{P}<0.05$ vs. the relative hyperfractionated group. The results are the means \pm SD of triplicate experiments. 

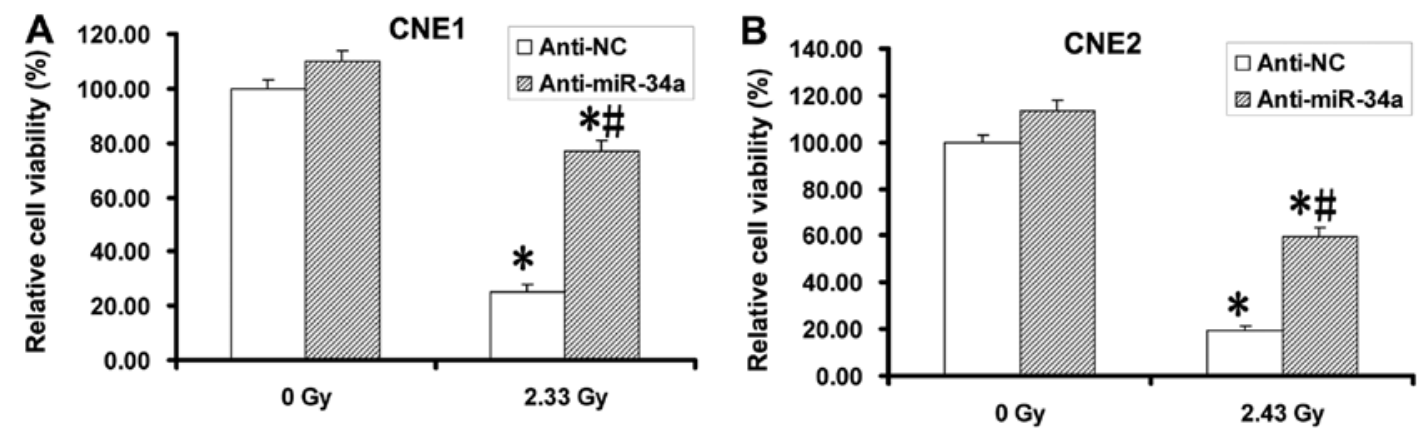

Figure 4. Knockdown of miR-34a significantly suppresses the growth inhibitory effects induced by hypofractionated radiotherapy. (A) CNE1 and (B) CNE2 cells were transfected with negative control inhibitor (anti-NC) or anti-miR-34a, and were than exposed to 2.33 Gy per fraction for 4 fractions, and $2.43 \mathrm{~Gy}$ per fraction for 4 fractions, respectively. Cell viability was detected by MTT assay, as described in "Materials and methods'. "P $<0.05$ vs. control. "P $<0.05$ vs. anti-NC. The results are the means \pm SD of triplicate experiments.

A

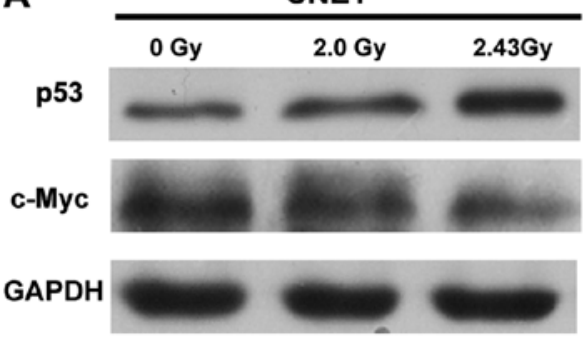

B

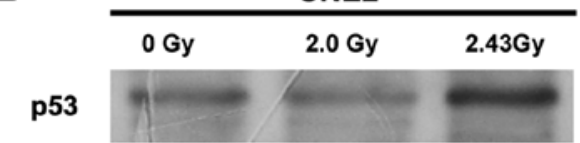

c-Myc

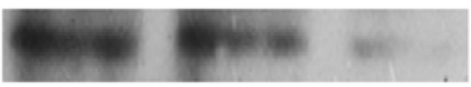

GAPDH

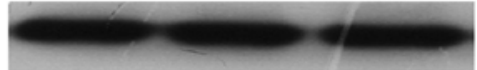

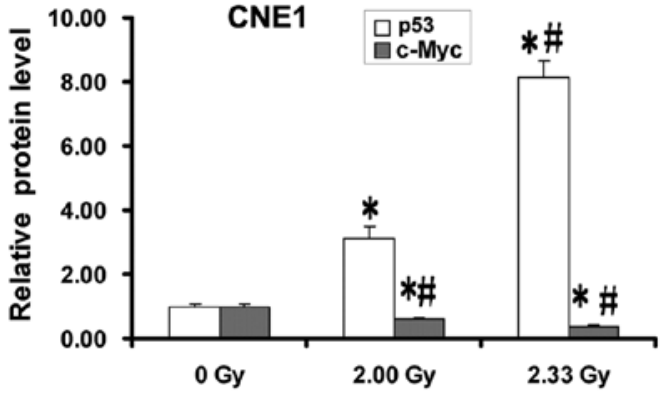

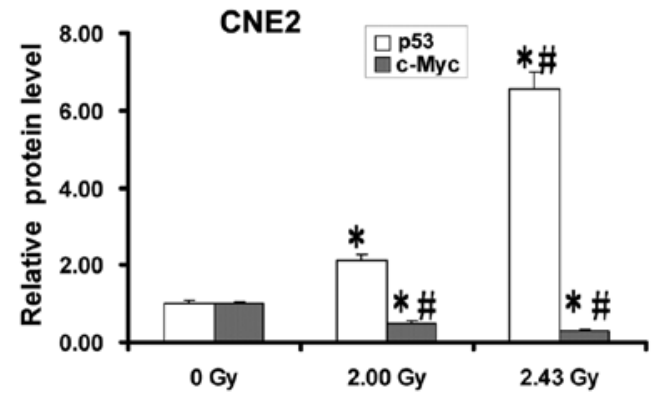

Figure 5. Hypofractionated radiotherapy enhances p53 protein expression and downregulates c-Myc protein expression. (A) CNE1 and (B) CNE2 cells were exposed to 2 Gy 6 MV X-ray per fraction for 5 fractions in the relative hyperfractionated group, and (A) CNE1 cells were exposed to 2.33 Gy per fraction for 4 fractions in the hypofractionated group, while (B) CNE2 cells were exposed to $2.43 \mathrm{~Gy}$ per fraction for 4 fractions in the hypofractionated group. The protein levels of $\mathrm{p} 53$ and c-Myc were then analyzed by western blot analysis. GAPDH was used to as the loading control. ${ }^{*} \mathrm{P}<0.05$ vs. control. ${ }^{*} \mathrm{P}<0.05$ vs. the relative hyperfractionated group. The results are the means $\pm \mathrm{SD}$ of triplicate experiments.
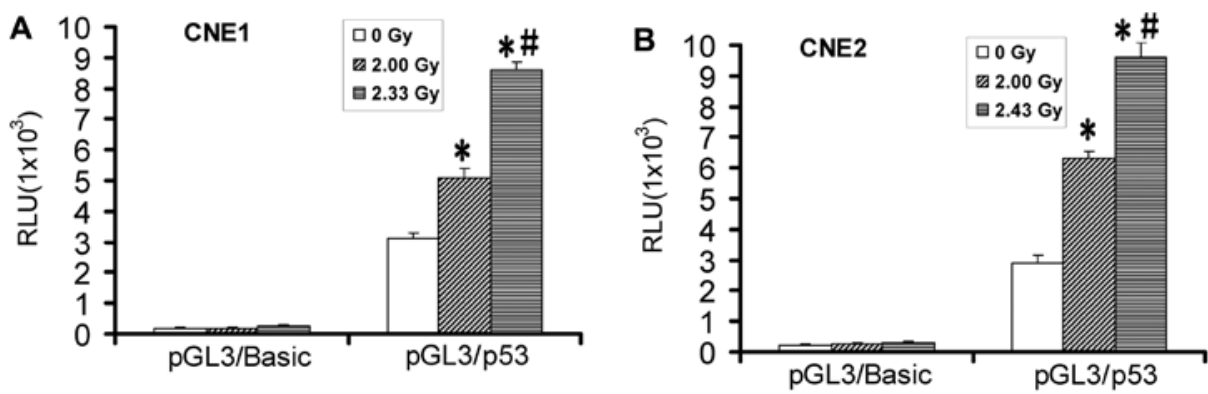

Figure 6. Hypofractionated radiotherapy enhances 553 promoter activity. (A) CNE1 and (B) CNE2 cells were transfected with pGL3/basic plasmid or the pGL3/p53 promoter plasmid, and were then exposed to $2 \mathrm{~Gy} 6 \mathrm{MV}$ X-ray per fraction for 5 fractions in the relative hyperfractionated group, and (A) CNE1 cells were exposed to $2.33 \mathrm{~Gy}$ per fraction for 4 fractions in the hypofractionated group, while (B) CNE2 cells were exposed to 2.43 Gy per fraction for 4 fractions in the hypofractionated group.The dual-luciferase reporter assay for $\mathrm{p} 53$ promoter activity was performed as described in 'Materials and methods'. "P $<0.05$ vs. control. "P<0.05 vs. the relative hyperfractionated group. The results are the means $\pm \mathrm{SD}$ of triplicate experiments. 
that miR-34a expression is induced through the p53-dependent pathway.

\section{Discussion}

In radiotherapy, the therapeutic efficacy in controlling cancer is often limited by tumor tissue radioresistance, including NPC radiotherapy (18). Although a more accurate tumor localization can be obtained by computed tomography, improved radiotherapy techniques and by combined radiochemotherapy in relatively advanced cases, which contributes to the improvement of the local control of the disease, radioresistance remains a serious obstacle in the successful treatment of the disease. Many patients present with local recurrence and distant metastases following radiotherapy (19). Therefore, there is an urgent need to explore the molecular mechanisms responsible for NPC radioresistance and to design more effective strategies for radiotherapy. Since the hypofractionated schema is being used more widely in the treatment of breast cancer $(20,21)$, prostate cancer (22), hepatocellular carcinoma (23), lung cancer (24), as well as NPC (5), and the rates of toxicity have not increased with hypofractionated regimens (25), in this study, we examined the growth inhibitory and apoptosis inducing effects of this schema in vitro. Our data demonstrated that hypofractionated radiotherapy inhibited cell viability and induced the apoptosis of NPC cells more prominently than the relative groups treated with hyperfractionated radiotherapy, indicating that the hypofractionated schema is an effective strategy to overcome radioresistance and enhance the effects of radiotherapy. Since each type of cancer is biologically different from another $(26,27)$, it is important to measure the biological effects reliably when the dose-per-fraction or dose-rate is changed and to search for a more optimized fractionation radiation treatment scheme.

The transcription factor $\mathrm{p} 53$ is best known as a regulator of the cell cycle and apoptosis, which can be rapidly induced in response to a wide range of stresses, including DNA damage, hypoxia and oxidative damage (28). The p53-dependent signaling pathway has been reported to play an important role in the response to radiation, particularly in the sensitivity of different types of cancer cells to radiation (29-32). The analysis of the p53 pathway and its role in the response to radiotherapy in NPC patients may facilitate the identification of the patients' risk of poor disease outcome (33). The suppression of the p53-mediated apoptosis pathway contributes to radioresistance and the poor prognosis of patients with NPC (34). The tumor suppressor miRNA, miR-34a, which has been reported to be significantly downregulated in NPC (10), is also a critical regulator of the cellular response to DNA damage (11). Moreover, miR-34a has been suggested to be the direct target of p53 $(15,16,35)$, and the induction of miR-34a expression by radiotherapy has been demonstrated to occur in a p53-dependent manner $(12,14)$. In the present study, we demonstrated that hypofractionated radiotherapy significantly increased miR-34a expression in the NPC cells, and that the growth inhibitory effects were reversed by the knockdown of miR-34a with antimiR-34a inhibitor, suggesting that the upregulation of miR-34a expression indeed plays an important role in the effects of hypofractionated radiotherapy. Although miR-34a has also been demonstrated to be upregulated in response to low-dose, low-energy X-ray-induced DNA damage in breast cells (17), hypofractionated high-dose radiotherapy may exert a prominent enhanced effect on its expression, since the expression of miR-34a is possibly induced in a dose-dependent manner. In our study, both p53-dependent gene transcription and protein expression were upregulated following hypofractionated radiotherapy. Regarding the fact that the tumor suppressor gene p53 is mostly intact in NPC cells (36), miR-34a expression is possibly induced mainly through the p53-dependent pathway, although it may be upregulated by radiation and not by $\mathrm{p} 53$ alone (14).

It has been demonstrated that miRNA expression is mediated by 533 and that the induction of miRNA expression contributes to the downregulation of proteins observed following the activation of p53 by DNA damaging agents/radiation, which directly mediates its diverse tumor suppressive effects (37). Myc oncogenic transcription factors are activated in a large range of human tumors, and it has been estimated that over 100,000 deaths each year are related to the deregulation of Myc expression (38). Myc is required to maintain the malignant state, and the forced expression of Myc is sufficient to provoke tumor formation in mouse models of human cancer (38). Myc inactivation or suppression usually provokes rapid tumor regression (39). miR-34a has been reported to attenuate the expression of c-Myc by directly targeting the $3^{\prime}$ UTR of its mRNA in a p53-dependent manner $(16,40)$. miR-34a also plays a novel role in the regulation of the transcription by c-Myc by suppressing the formation and function of the c-Myc complex that activates or elongates transcription (40). In the present study, c-Myc protein expression was markedly downregulated by hypofractionated radiotherapy, strongly suggesting that the enhanced apoptosis of NPC cells is associated with the miR-34a-mediated suppression of c-Myc in a p53-dependent manner. In addition, Bcl-2 and Notch-1, which also play a critical role in cell fate decisions by modulating cellular processes under irradiation, have been demonstrated to be inhibited by miR-34a in a p53-dependent manner $(12,13)$. Further studies are required in these areas to further illuminate the role of these proteins in the effects of hypofractionated radiotherapy.

In conclusion, the present study demonstrates that hypofractionated radiotherapy induces miR-34a expression, downregulates c-Myc protein expression and enhances the apoptosis of human NPC cells probably through the p53-dependent pathway. Moreover, we our data demonstrate that the upregulated expression of miR-34a indeed plays a critical role in the hypofractionated schema. The present study indicates that the hypofractionated schema is an effective strategy to enhance the effects of radiotherapy on human NPC cells.

\section{References}

1. Orecchia R, Redda MG, Ragona R, et al: Results of hypofractionated stereotactic re-irradiation on 13 locally recurrent nasopharyngeal carcinomas. Radiother Oncol 53: 23-28, 1999.

2. Pan Y, Wang M, Bu X, et al: Curcumin analogue T83 exhibits potent antitumor activity and induces radiosensitivity through inactivation of Jab1 in nasopharyngeal carcinoma. BMC Cancer 13: 323, 2013.

3. Wu JS, Brasher PM, El-Gayed A, et al: Phase II study of hypofractionated image-guided radiotherapy for localized prostate cancer: outcomes of $55 \mathrm{~Gy}$ in 16 fractions at $3.4 \mathrm{~Gy}$ per fraction. Radiother Oncol 103: 210-216, 2012. 
4. Schneider U, Besserer J and Mack A: Hypofractionated radiotherapy has the potential for second cancer reduction. Theor Biol Med Model 7: 4, 2010.

5. Bakst RL, Lee N, Pfister DG, et al: Hypofractionated dose-painting intensity modulated radiation therapy with chemotherapy for nasopharyngeal carcinoma: a prospective trial. Int J Radiat Oncol Biol Phys 80: 148-153, 2011.

6. Friedman JM, Jones PA and Liang G: The tumor suppressor microRNA-101 becomes an epigenetic player by targeting the polycomb group protein EZH2 in cancer. Cell Cycle 8: 2313-2314, 2009.

7. Jiang P, Rao EY, Meng N, Zhao Y and Wang JJ: MicroRNA-17-92 significantly enhances radioresistance in human mantle cell lymphoma cells. Radiat Oncol 5: 100, 2010.

8. Welch C, Chen Y and Stallings RL: MicroRNA-34a functions as a potential tumor suppressor by inducing apoptosis in neuroblastoma cells. Oncogene 26: 5017-5022, 2007.

9. Liu X, Luo HN, Tian WD, et al: Diagnostic and prognostic value of plasma microRNA deregulation in nasopharyngeal carcinoma. Cancer Biol Ther 14: 1133-1142, 2013.

10. Chen HC, Chen GH, Chen YH, et al: MicroRNA deregulation and pathway alterations in nasopharyngeal carcinoma. $\mathrm{Br}$ J Cancer 100: 1002-1011, 2009.

11. Ghawanmeh T, Thunberg U, Castro J, Murray $F$ and Laytragoon-Lewin N: miR-34a expression, cell cycle arrest and cell death of malignant mesothelioma cells upon treatment with radiation, docetaxel or combination treatment. Oncology 81: 330-335, 2011

12. Liu C, Zhou C, Gao F, et al: MiR-34a in age and tissue related radio-sensitivity and serum miR-34a as a novel indicator of radiation injury. Int J Biol Sci 7: 221-233, 2011.

13. Kang J, Kim E, Kim W, et al: Rhamnetin and cirsiliol induce radiosensitization and inhibition of epithelial-mesenchymal transition (EMT) by miR-34a-mediated suppression of Notch-1 expression in non-small cell lung cancer cell lines. J Biol Chem 288: 27343-27357, 2013.

14. John-Aryankalayil M, Palayoor ST, Makinde AY, et al: Fractionated radiation alters oncomir and tumor suppressor miRNAs in human prostate cancer cells. Radiat Res 178: 105-117, 2012.

15. Boysen J, Sinha S, Price-Troska T, et al: The tumor suppressor axis $\mathrm{p} 53 / \mathrm{miR}-34$ a regulates Axl expression in B-cell chronic lymphocytic leukemia: implications for therapy in p53-defective CLL patients. Leukemia 28: 451-455, 2013.

16. Su W, Hopkins S, Nesser NK, et al: The p53 transcription factor modulates microglia behavior through microRNA-dependent regulation of c-Maf. J Immunol 92: 358-366, 2013.

17. Stankevicins L, Almeida da Silva AP, Ventura Dos Passos F, et al: MiR-34a is up-regulated in response to low dose, low energy X-ray induced DNA damage in breast cells. Radiat Oncol 8: 231, 2013.

18. Qu Y, Zhao S, Hong J and Tang S: Radiosensitive gene therapy through imRNA expression for silencing manganese superoxide dismutase. J Cancer Res Clin Oncol 136: 953-959, 2010.

19. Feng XP, Yi H, Li MY, et al: Identification of biomarkers for predicting nasopharyngeal carcinoma response to radiotherapy by proteomics. Cancer Res 70: 3450-3462, 2010.

20. Bourgier C, Lemanski C, Riou O, Charissoux M, Fenoglietto $P$ and Azria D: Hypofractionated radiotherapy, a standard in breast cancer? Cancer Radiother 17: 705-707, 2013 (In French).

21. Tortorelli G, Di Murro L, Barbarino R, et al: Standard or hypofractionated radiotherapy in the postoperative treatment of breast cancer: a retrospective analysis of acute skin toxicity and dose inhomogeneities. BMC Cancer 13: 230, 2013.

22. Ishiyama H, Satoh T, Kitano M, et al: High-dose-rate brachytherapy and hypofractionated external beam radiotherapy combined with long-term hormonal therapy for high-risk and very high-risk prostate cancer: outcomes after 5-year follow-up. J Radiat Res 55: 509-517, 2013.
23. Huang BS, Tsang NM, Lin SM, et al: High-dose hypofractionated X-ray radiotherapy for hepatocellular carcinoma: Tumor responses and toxicities. Oncol Lett 6: 1514-1520, 2013.

24. Bush DA, Cheek G, Zaheer S, et al: High-dose hypofractionated proton beam radiation therapy is safe and effective for central and peripheral early-stage non-small cell lung cancer: results of a 12-year experience at Loma Linda University Medical Center. Int J Radiat Oncol Biol Phys 86: 964-968, 2013.

25. Mouw KW and Harris JR: Hypofractionation in the era of modulated radiotherapy (RT). Breast 22 (Suppl 2): S129-S136, 2013.

26. Fowler JF: The radiobiology of prostate cancer including new aspects of fractionated radiotherapy. Acta Oncol 44: 265-276, 2005.

27. Debenham BJ, Hu KS and Harrison LB: Present status and future directions of intraoperative radiotherapy. Lancet Oncol 14: e457-e464, 2013.

28. Mendrysa SM, Ghassemifar S and Malek R: p53 in the CNS: perspectives on development, stem cells, and cancer. Genes Cancer 2: 431-442, 2011.

29. Muaddi H, Chowdhury S, Vellanki R, Zamiara $P$ and Koritzinsky M: Contributions of AMPK and p53 dependent signaling to radiation response in the presence of metformin. Radiother Oncol 108: 446-450, 2013.

30. Huerta S, Gao X, Dineen S, Kapur P, Saha D and Meyer J: Role of p53, Bax, p21, and DNA-PKes in radiation sensitivity of HCT-116 cells and xenografts. Surgery 154: 143-151, 2013.

31. Gilbert DC, Williams A, Allan K, et al: p16 ${ }^{\mathrm{INK} 4 \mathrm{~A}}$, p53, EGFR expression and KRAS mutation status in squamous cell cancers of the anus: correlation with outcomes following chemo-radiotherapy. Radiother Oncol 109: 146-151, 2013.

32. Guo Y, Parry JJ, Laforest R, Rogers BE and Anderson CJ: The role of p53 in combination radioimmunotherapy with $64 \mathrm{Cu}$-DOTAcetuximab and cisplatin in a mouse model of colorectal cancer. J Nucl Med 54: 1621-1629, 2013.

33. Xie X, Jin $\mathrm{H}, \mathrm{Hu}$ J, et al: Association between single nucleotide polymorphisms in the p53 pathway and response to radiotherapy in patients with nasopharyngeal carcinoma. Oncol Rep 31: 223-231, 2014

34. Yang CF, Peng LX, Huang TJ, et al: Cancer stem-like cell characteristics induced by EB virus-encoded LMP1 contribute to radioresistance in nasopharyngeal carcinoma by suppressing the p53-mediated apoptosis pathway. Cancer Lett 344: 260-271, 2013.

35. Siemens H, Jackstadt R, Kaller M and Hermeking H: Repression of c-Kit by p53 is mediated by miR-34 and is associated with reduced chemoresistance, migration and stemness. Oncotarget 4: 1399-1415, 2013.

36. Yoshizaki T, Ito M, Murono S, Wakisaka N, Kondo S and Endo K: Current understanding and management of nasopharyngeal carcinoma. Auris Nasus Larynx 39: 137-144, 2012.

37. Hermeking H: The miR-34 family in cancer and apoptosis. Cell Death Differ 17: 193-199,2010.

38. Jain M, Arvanitis C, Chu K, et al: Sustained loss of a neoplastic phenotype by brief inactivation of MYC. Science 297: 102-104, 2002.

39. Doherty JR, Yang C, Scott KE, et al: Blocking lactate export by inhibiting the Myc target MCT1 disables glycolysis and glutathione synthesis. Cancer Res 74: 908-920, 2013.

40. Yamamura S, Saini S, Majid S, et al: MicroRNA-34a suppresses malignant transformation by targeting c-Myc transcriptional complexes in human renal cell carcinoma. Carcinogenesis 33: 294-300, 2012 